\title{
Vector competence of Aedes aegypti and screening for differentially expressed microRNAs exposed to Zika virus
}

\author{
Chunling Zhu ${ }^{1,2+}$, Yuting Jiang ${ }^{1+}{ }^{-0}$, Qianghui Zhang ${ }^{1}$, Jian Gao ${ }^{1}$, Chaojie $\mathrm{Li}^{1}$, Chunxiao $\mathrm{Li}^{1}$, Yande Dong ${ }^{1}$, \\ Dan Xing ${ }^{1}$, Hengduan Zhang ${ }^{1}$, Teng Zhao ${ }^{1}$, Xiaoxia Guo ${ }^{1 *}$ and Tongyan Zhao ${ }^{1 *}$
}

\begin{abstract}
Background: Zika virus (ZIKV) is transmitted to humans primarily by Aedes aegypti. Previous studies on Ae. aegypti from Jiegao (JG) and Mengding (MD) in Yunnan province, China have shown that these mosquitoes are able to transmit ZIKV to their offspring through vertical transmission, indicating that these two Ae. aegypti strains pose a potential risk for ZIKV transmission. However, the vector competence of these two Ae. aegypti strains to ZIKV has not been evaluated and the molecular mechanisms influencing vector competence are still unclear.
\end{abstract}

Methods: Aedes aegypti mosquitoes from JG and MD were orally infected with ZIKV, and the infection rate (IR), dissemination rate (DR), transmission rate (TR) and transmission efficiency (TE) of these two mosquito strains were explored to evaluate their vector competence to ZIKV. On 2, 4 and 6 days post-infection (dpi), the small RNA profiles between ZIKV-infected and non-infected Ae. aegypti midgut and salivary gland tissues were compared to gain insights into the molecular interactions between ZIKV and Ae. aegypti.

Results: There were no significant differences in the IR, DR, TR and TE between the two Ae. aegypti strains $(P>0.05)$. However, ZIKV RNA appeared 2 days earlier in saliva of the JG strain, which indicated a higher competence of the JG strain to transmit ZIKV. Significant differences in the microRNA (miRNA) expression profiles between ZIKV-infected and non-infected Ae. aegypti were found in the 2-dpi libraries of both the midgut and salivary gland tissues from the two strains. In addition, 27 and 74 miRNAs ( $\mid$ log2 fold change| > 2) were selected from the miRNA expression profiles of ZIKV-infected and non-infected midgut and salivary gland tissues from the JG and MD strains, respectively.

Conclusions: Our results provide novel insights into the ZIKV-mosquito interactions and build a foundation for future research on how miRNAs regulate the vector competence of mosquitoes to this arbovirus.

Keywords: Zika virus, Aedes aegypti, Vector competence, microRNA

*Correspondence: guoxx99@163.com; tongyanzhao@126.com

${ }^{\dagger}$ Chunling Zhu and Yuting Jiang contributed equally to this work

1 Department of Vector Biology and Control, State Key Laboratory of Pathogen and Biosecurity, Beijing Key Laboratory of Vector Borne and Natural Focus Infectious Diseases, Beijing Institute of Microbiology and Epidemiology, Beijing 100071, China

Full list of author information is available at the end of the article

\section{Background}

Zika virus (ZIKV) (family Flaviviridae, genus Flavivirus) is a single-stranded RNA virus that is primarily transmitted by Aedes aegypti. ZIKV was discovered initially from a sentinel monkey in Uganda, Africa, in 1947 [1, 2] and has since diverged into two main genotypes: African and Asian genotypes [3]. The first case of human infection in Brazil was recorded in 2015 [4, 5]. ZIKV continues to spread, currently recorded in 86 countries original author(s) and the source, provide a link to the Creative Commons licence, and indicate if changes were made. The images or other third party material in this article are included in the article's Creative Commons licence, unless indicated otherwise in a credit line to the material. If material is not included in the article's Creative Commons licence and your intended use is not permitted by statutory regulation or exceeds the permitted use, you will need to obtain permission directly from the copyright holder. To view a copy of this licence, visit http://creativecommons.org/licenses/by/4.0/. The Creative Commons Public Domain Dedication waiver (http://creativeco mmons.org/publicdomain/zero/1.0/) applies to the data made available in this article, unless otherwise stated in a credit line to the data. 
or regions around the world, and it is estimated that approximately 3.6 billion people currently live in areas at risk of transmission [6]. ZIKV can cause Guillain-Barré syndrome (an acute inflammatory immune-mediated polyneuropathy) in adults and fetal microcephaly in newborns [7]. The spread of ZIKV in the Western Hemisphere poses a major threat to public health. Currently, there are no specific drugs or vaccines against ZIKV infection, with vector control being the only viable method for alleviating the diseases caused by this virus. Genetic manipulation of vectors to modulate vector competence is also a potential novel approach for controlling vector-borne diseases [8-10]. Therefore, knowledge of molecular interactions between viruses and mosquito hosts is critical to manipulating vector competence.

Vector competence to arboviruses is affected by both intrinsic factors and the molecular mechanism in mosquitoes. When a female mosquito ingests an infectious blood meal, the first organ that an arbovirus comes into contact with is the midgut of the mosquito. The arbovirus then enters the hemolymph and spreads to secondary tissues, such as the fat body, trachea and salivary gland. Finally, the virus is released into salivary tubes and transmitted to uninfected vertebrate hosts by horizontal transmission [11]. Therefore, in order to infect a vector, the virus must overcome the tissue barriers associated with the midgut and salivary gland and cope with the related antiviral pathways. In this context, exploration of the interactions of virus and tissue barriers to arbovirus infection may provide interesting results.

Mosquitoes possess a few antiviral pathways that are able to limit the spread of virus, such as reactive oxygen species (ROS) production and the innate immune pathway. However, these approaches appear to be virus specific. It has been shown that the antioxidants in the midgut induced by catalase are triggered after the mosquito has ingested a blood meal and that this state of antioxidation can increase mosquito susceptibility to dengue virus (DENV), but does not affect ZIKV infection [12]. In the mosquito's innate immune pathways, the Toll and JAK-STAT pathways are related to the regulation of DENV infection, yet they do not appear to influence other arboviruses, such as chikungunya virus (CHIKV) or ZIKV [13-16]. The RNA interference (RNAi) pathway is also an important way to determine the interactions between arboviruses and their mosquito hosts. The RNAi pathway mainly includes three main small RNA pathways: small interfering RNA (siRNA), microRNA (miRNA) and P-element-induced wimpy testisinteracting RNA (piRNA) [17]. Although miRNAs could potentially be involved in ZIKV-mosquito interactions, the direct interaction and specific mechanism are still unclear [18].
miRNAs are endogenous small non-coding RNAs of 21-24 nucleotides (nt) that regulate gene expression through binding to the target mRNA and initiating mRNA degradation by the RNA-induced silencing complex (RISC) [19]. In mosquitoes, miRNAs are involved in the replication of virus by regulating host factors. Some studies have indicated that DENV [20] and ZIKV [18] infections could alter miRNA profiles in mosquitoes. The possibility that some miRNAs in Aedes may affect viral replication has also been reported. In one study, the miRNA miR-252 in Ae. albopictus increased after a DENV-infected blood meal, and inhibition of the miRNA enhanced viral replication while overexpression of the miRNA limited viral replication [21]. Another study demonstrated that aae-miR-375 could enhance DENV serotype 2 infection in an Ae. aegypti cell line [22]. These studies indicate that miRNAs play an important regulatory role in the interactions between arboviruses and mosquitoes, which may affect the vector competence of mosquitoes. It is possible that more obvious changes in miRNAs could be observed in specific tissues infected with virus.

Aedes aegypti is an important invasive species in Yunnan province in China since 2002 [23]. Yunnan province is the main passageway for China to connect to Southeast Asia, and Jiegao and Mengding are two important trade ports in this province. To date, there has been no study on the risk of a ZIKV epidemic through mosquitoes in these two cities. In the present study, we collected two Ae. aegypti strains in Jiegao and Mengding to assess their vector competence to ZIKV. We also analyzed the results of high-throughput sequencing of microRNAs in the midgut and salivary gland infected with ZIKV to explore the molecular interactions between the mosquito host and the virus, which could contribute to development of new insect-borne disease prevention strategies.

\section{Methods \\ Virus strain}

The viral strain used in this study was ZIKV SZ01 strain obtained from the Microbial Culture Collection Center of the Beijing Institute of Microbiology and Epidemiology. This virus was originally isolated from a patient who returned from Samoa to China in 2016 (GenBank Nos. KU866423) [24]. The virus has been maintained in the Aedes albopictus C6/36 cell lines and was passaged four times before the study.

\section{Infection of mosquitoes}

Mosquito larvae and pupae of two Ae. aegypti mosquito strains were originally collected from Jiegao $\left(23^{\circ} 58^{\prime} 40^{\prime \prime} \mathrm{N}\right.$, $\left.97^{\circ} 53^{\prime} 24^{\prime \prime} \mathrm{E}\right)$ and Mengding $\left(23^{\circ} 33^{\prime} 00^{\prime \prime} \mathrm{N}, 9^{\circ} 3^{\prime} 33^{\prime \prime} \mathrm{E}\right)$, Yunnan Province, China, respectively, in July 2018. All larvae 
and pupae were reared to adult forms in the laboratory under the same conditions $\left(26 \pm 1{ }^{\circ} \mathrm{C}\right.$, relative humidity [RH] $75 \pm 5 \%$, a 14:10-h light/dark [L/D] photoperiod). Adult mosquitoes were provided with $10 \%$ sugar water. The fourth generation of two Ae. aegypti stains were used to perform ZIKV oral infection experiments. Detailed methods of mosquito collection and husbandry are described in our previous work [25].

ZIKV suspension mixed with mouse blood (1:1) were provided to 5-day-old female mosquitoes for oral feeding using the Hemotek membrane feeding system (Sihuan, Beijing, China) to keep the virus blood meal at $37^{\circ} \mathrm{C}$. The virus titer of the virus blood meal was determined to be $1.5 \times 10^{4}$ plaque-forming units $(\mathrm{PFU}) / \mathrm{ml}$ at the time of feeding, using the plaque assay with the BHK-21 cell line (ATCC Number: CCL-10) . After $1 \mathrm{~h}$ of blood-feeding, fully engorged females were transferred to plastic cups (10-15 mosquitoes/tube) and reared at $29 \pm 1{ }^{\circ} \mathrm{C}$ and $75 \pm 5 \% \mathrm{RH}$ under a $14 / 10$-h L/D cycle. A $5 \%$ sucrose solution on cotton pads was provided to these mosquitoes.

\section{Vector competence indices}

For each mosquito examined, the midgut, salivary gland and saliva were tested separately to analyze vector competence in the two Ae. aegypti strains at 2, 4, 6, 8, 10, 14 and 20 days post-infection (dpi), and 10 mosquitoes were used per time point. The experiments were performed three times independently, except for those carried out on $20 \mathrm{dpi}$ that were performed two times. The midgut and salivary gland of each mosquito were dissected and washed 3 times in phosphate-buffered saline and transferred to $1.5-\mathrm{ml}$ microtubes containing $100 \mu \mathrm{l}$ of Dulbecco's modified Eagle's medium (DMEM; GIBCO ${ }^{\mathrm{TM}}$, Invitrogen, Beijing, China) supplemented with $2 \%$ fetal bovine serum (FBS). Saliva was collected from individual mosquitoes at $2,4,6,8,10,14$ and 20 dpi using the method described by Dubrulle et al. [26]. After removing the wings and legs of each mosquito, the proboscis was inserted into a glass capillary containing $5 \mu$ of FBS. After 30 min, the FBS containing saliva was mixed with $100 \mu \mathrm{l}$ of DMEM in $1.5-\mathrm{ml}$ microtubes.

The ZIKA RNA of these samples was extracted using the QIAamp Viral RNA Mini kit (Qiagen, Hilden, Germany) and detected using a commercial kit (Zika virus nucleic acid detection kit; Daan Gene Co. Ltd., Guangdong, China; Cat no: DA0711). The procedures are described in detail in our previous study [25].

Vector competence of the mosquitoes was evaluated by calculating the infection rate (IR; number of positive midgut/the total number of mosquitoes tested), dissemination rate (DR; number of infected salivary gland/ the number of infected midgut), transmission rate (TR; number of infected saliva/the number of infected salivary gland) and transmission efficiency (TE; number of infected saliva/the total number of mosquitoes tested).

All statistical analyses were conducted using SPSS version 21.0 software (SPSS IBM Corp., Armonk, NY, USA). The IR, DR, TR and TE of two Ae. aegypti strains were compared using Fisher's exact test. Viral titers were compared with the Mann-Whitney U-test to determine differences between two strains at different times. $P$-values $<0.05$ were considered to be significant.

\section{Viability of ZIKV in saliva of Ae. aegypti}

Saliva samples were collected from JG and MD Ae. aegypti strains (30 individuals of each strain) on $8 \mathrm{dpi}$ using the method described by Dubrulle et al. [26]. In brief, wings and legs of each mosquito were removed and the proboscis was inserted into a glass capillary containing $5 \mu \mathrm{l}$ of FBS. After 30 min of salivation, FBS containing saliva collected from 30 individual mosquitoes was

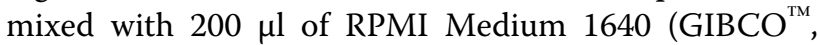
Invitrogen, Beijing, China). The mixture was then inoculated into one well of a 12-well plate covered with a single layer of C6/36 cells for $2 \mathrm{~h}$. After the incubation period, the mixture was removed and $1 \mathrm{ml}$ RPMI Medium 1640 (containing 2\% FBS, 1\% 100U/ml penicillin and $100 \mathrm{ug} /$ $\mathrm{ml}$ streptomycin) was added into the well. The plate was maintained in a cell incubator at $28{ }^{\circ} \mathrm{C}$ and $5 \% \mathrm{CO}_{2}$ for 7 days. Cells were observed by inverted microscopy every day to examine the cytopathic effect (CPE).

\section{Sample preparation and RNA extraction}

Five-day-old female mosquitoes from the two strains (JG and MD) were fed with ZIKV-infected blood meal $\left(1.5 \times 10^{4} \mathrm{PFU} / \mathrm{ml}\right)$ or a blood meal devoid of ZIKV. The infection procedure was the same as described for the vector competence test. The midgut and salivary gland of approximately 100 mosquitoes from each of the groups (infected group and control group) of the two strains were collected by dissecting individual mosquitoes on 2 , 4 and $6 \mathrm{dpi}$. These samples were collected separately in $1.5-\mathrm{ml}$ RNase-free microcentrifuge tubes containing 500 $\mu \mathrm{l}$ TRIzol reagent (Invitrogen, Thermo Fisher Scientific, Waltham, MA USA) and stored at $-80^{\circ} \mathrm{C}$ until subsequent RNA extraction. Total RNA was extracted from 24 groups using the TRIzol reagent according to the manufacturer's protocol. The quality and quantity of RNA were measured by the Agilent 2100 Bioanalyzer System (Agilent Technologies, Inc., Santa Clara, CA, USA). Each RNA sample was divided to two parts, with one used for small RNA library preparation and sequencing and the second part used for reverse transcription-quantitative PCR (RT-qPCR) analysis. 


\section{Small RNA library preparation and sequencing}

The RNA extraction, library preparation and sequencing analyses were performed by the BGI Company (Shenzhen, China). Briefly, $1 \mu \mathrm{g}$ of each pooled total RNA was used to create small RNA libraries. RNA fragments (18-30 nt) were isolated from total RNA by polyacrylamide gel electrophoresis (PAGE). Small RNA libraries were prepared in accordance with the manufacturer's instructions (Illumina, San Diego, CA, USA). Library creation uses a sequential addition of first a $3^{\prime}$ adapter sequence followed by a $5^{\prime}$ adapter sequence. A complimentary DNA (cDNA) copy was then synthesized using ProtoScript reverse transcriptase (New England Biolabs, Ipswich, MA, USA) and a primer complimentary to a segment of the $3^{\prime}$ adapter. The cDNA was amplified using reverse transcriptase PCR (RT-PCR) for 12-15 PCR cycles to complete the libraries. The quality of cDNA was checked using the Agilent 2100 Bioanalyzer system (Agilent Technologies Inc.). Libraries were then submitted to BGI (Shenzhen, China) for small RNA sequencing (RNAseq) using the Illumina genomic analyzer.

\section{Bioinformatics}

The Fastx toolkit was used to remove adapter sequences and reads with low-quality scores from datasets; Bowtie2 was used to map clean reads to the reference genome and to other sRNA databases; and cmsearch was used for searching the Rfam database. After removing the adapter and low-quality sequences, all clean small RNA tags were matched with the NCBI GenBank (http://www.ncbi.nlm. gov/) ribosome RNA (rRNA), small cytoplasmic RNA (scRNA, small nucleolar RNA (snoRNA), small nuclear RNA (snRNA) and transfer RNA (tRNA) databases, and the matched tags were removed from the unlabeled tags. In order to ensure that every small RNA mapped to only one annotation, small RNA was classified and annotated according to the priority rule of all rRNA (in which GenBank $>$ Rfam $>$ repeat $>$ exon $>$ intron $>$ known miRNA) [27]. Mature and pre-miRNA sequences of Ae. aegypti (AaegL5.0) were used as a reference miRNA from the miRBase v.21 database. The unmatched clean reads were used for the prediction of novel miRNAs by MiRDeep2 by exploring their secondary structure, information of Dicer cleavage sites and energy. RNA secondary structures were predicted by RNA-fold (http://rna.tbi.univie. ac.at/).

\section{Expression profiling of miRNAs in response to ZIKV}

An algorithm was used to identify differentially expressed miRNAs between ZIKV-infected samples and control samples. $p(\mathrm{X})=\mathrm{e}^{-\lambda} \lambda^{\mathrm{X}} / \mathrm{X}$ !, where $\mathrm{X}$ is defined as the number of reads from small RNA and $\lambda$ is the real transcripts of the miRNA. The method is described in detail by
Audic et al. [28]. When the false discovery rate (FDR) was $<0.001$, changes in known miRNA expression between infected samples and control samples were considered to be significant. miRNAs with $\log 2$ fold change $(\mathrm{FC})>1$ were designated as being significantly upregulated, and miRNAs with $\log 2 \mathrm{FC} \leq 1$ were designated as being significantly downregulated.

\section{RT-qPCR analysis of miRNAs}

The expression of miRNAs was validated by two-step RT-qPCR using the primers listed in Additional file 1: Table S1. The RT reaction was conducted in a mixture containing $2 \mu \mathrm{l}$ of dNTP ( $2.5 \mathrm{mM}$ each), $2 \mu \mathrm{l}$ of $10 \times \mathrm{RT}$ Buffer, $2 \mu \mathrm{l}$ of RT primer $(10 \mu \mathrm{M}), 8 \mu \mathrm{l}$ of total RNA, $1 \mu \mathrm{l}$ of RT enzyme (10 U/ul), $1 \mu \mathrm{l}$ of RNase inhibitor $(40 \mathrm{u} / \mathrm{ul})$ and $4 \mu \mathrm{l}$ of RNase free water, at $16^{\circ} \mathrm{C}$ for $30 \mathrm{~min}, 42{ }^{\circ} \mathrm{C}$ for $40 \mathrm{~min}$ and $85^{\circ} \mathrm{C}$ for $5 \mathrm{~min}$. All RT reagents were purchased from Invitrogen (Carlsbad, CA, USA). The resulting cDNAs were used as templates for the qPCR reaction, which contained $8 \mu \mathrm{l}$ of $2 \times$ PCR mix (Lot: 204145; Qiagen), $0.2 \mu \mathrm{l}$ of each forward and reverse primers $(10 \mu \mathrm{M})$, $1 \mu \mathrm{l}$ of template and $6.6 \mu \mathrm{l}$ of nuclease water. Amplification reactions were performed in an ABI ViiA 7 RealTime PCR System (Applied Biosystems, Thermo Fisher Scientific, Waltham, MA, USA) programed as follows: 1 cycle at $95^{\circ} \mathrm{C}$ for $2 \mathrm{~min}$; then $94^{\circ} \mathrm{C}$ for $10 \mathrm{~s}$ and 40 cycles at $55^{\circ} \mathrm{C}$ for $10 \mathrm{~s}$ and $72{ }^{\circ} \mathrm{C}$ for $40 \mathrm{~s}$. The analysis of relative expression levels of miRNAs was performed using $2^{-\Delta \Delta C T}$ method [29]. Small nuclear RNA (RNU6B) was used to normalize the FC in miRNA expression in each sample.

\section{Target prediction of miRNAs and functional analysis}

The target genes of the differentially expressed miRNA were predicted using miRanda (http://www.microrna. org/microrna/home.do) and RNAhybrid (http://bibis erv.techfak.uni-bielefeld.de/rnahybrid/). The prediction of the functions of these miRNAs or their targets based on Gene Ontology (GO; http://amigo.geneontolo gy.org/amigo) and the Kyoto Encyclopedia of Genes and Genomes (KEGG; https://www.genome.jp/kegg/pathway. html) pathway enrichment analysis.

\section{Results}

\section{Vector competence to ZIKV in two Ae. aegypti strains}

To evaluate the vector competence of Ae. aegypti (JG and MD strains) to ZIKV, we assessed the IR, DR, TR and TE. ZIKV RNA copies in the mosquito midgut, salivary gland and saliva were detected on 2, 4, 6, 8, 10, 14 and 20 dpi. ZIKV particles secreted in saliva were detected on $8 \mathrm{dpi}$. From 2 dpi onward, the midgut and salivary gland of each mosquito strain tested positive for ZIKV RNA. Overall, infection rates were moderate $(40-60 \%$ for JG, $25-50 \%$ 
for $\mathrm{MD}$ ). The $\mathrm{DR}$ were around $27-75 \%$ in the JG strain and $33-90 \%$ in the MD strain from 2 to $20 \mathrm{dpi}$, increasing with increasing time from initial infection. Between 14 and $20 \mathrm{dpi}$, the DR and TR remained high in the two Ae. aegypti strains (DR: 75-80\%, TR: 55-125\%). The transmission efficiencies of the two Ae. aegypti strains ranged from 7 to $25 \%$. There were no significant differences in the IR, DR, TR and TE between the two $A e$. aegypti strains at any time point during the experiments (Fisher's exact test, $P=0.179 \sim 1.000$; Fig. 1a). ZIKV RNA appeared 2 days earlier in saliva of the JG strain compared to the MD strain [ 4 vs 6 dpi; Fig. 1a (iv)], which indicates a higher vector competence of the JG strain to ZIKV.

The number of ZIKV RNA copies (log10 transformation) increased in the midgut and salivary gland of each mosquito strain over time, reaching $5.17 \pm 0.89$ and $4.72 \pm 0.52$ in these two tissues on $20 \mathrm{dpi}$, respectively. Virus level was low in the saliva and remained between 2.35 and 3.12 during $6-20 \mathrm{dpi}$. There was no significant difference in virus RNA level between two strains at any time point during the experiments (Mann-Whitney U-test, $U=12.50 \sim 22.00, P=0.125 \sim 0.748$; Fig. 1b).

On 8 dpi, saliva samples collected from the ZIKAinfected JG and MD Ae. aegypti strains (30 individuals of each strain) were inoculated separately into C6/36 cells, and uninfected C6/36 cells were used as the control (Fig. 1c). On day 7, the CPE could be observed in the saliva cells inoculated with infected saliva but not in the control group.

\section{Illumina sequencing of small RNAs}

An Illumina sequencing platform was used to produce small RNA profiles of ZIKV-infected and non-infected Ae. aegypti mosquitoes (JG and $\mathrm{MD}$ strains, midgut and salivary gland). For the JG strain, we obtained 178 and 181 million combined raw reads from control and infected small RNA libraries, respectively; for the MD strain, 182 and 176 million reads were acquired from control and infected libraries, respectively (Additional file 2: Table S2). In order to obtain the clean reads, 9-33\% of reads were discarded in different libraries due to their low-quality score or lack of adapter sequence. In those libraries, the small RNA length distribution showed a peak at 21-23 nt, representing the characteristic lengths of miRNAs and siRNAs. Another smaller peak was obtained at 27-30 nt, which may be related to piRNAs, a common feature of insect small RNA libraries (Fig. 2).

\section{Changes in Ae. aegypti miRNA expression profiles in response to ZIKV infection}

Small RNA library analysis of ZIKV-infected Ae. aegypti midgut and salivary gland tissues identified 87 differentially regulated miRNAs for the JG strain and 216 differentially regulated miRNAs for the MD strain at the different time points compared with non-infected controls $(|\log 2 \mathrm{FC}|>1)$ (Fig. 3). Interestingly, the most alterations in the miRNA profile were found in the 2-dpi libraries of both tissues from two strains. In addition, 27 and 74 miRNAs were identified from the JG and MD strains, respectively, with a standard of $|\log 2 \mathrm{FC}|>2$ in expression between the ZIKV-infected and non-infected group. (Additional file 3: Table S3). Of these miRNAs, eight were upregulated and six were downregulated with statistical significance in the midgut tissue, and three were upregulated and 10 were downregulated with statistical significance in the salivary gland tissue of JG strain. However, the changes in miRNA expression in the midgut and salivary gland tissues of MD strain were predominately upregulated.

There were three miRNAs (aae-miR-2946, aaemiR-989, aae-miR-2941) in the midgut with $|\log 2 \mathrm{FC}|>2$ expressed in both the JG and MD strains of Ae. aegypti mosquitoes (Fig. 4; Table 1). These three miRNAs were upregulated in the midgut of the MD strain but downregulated in the midgut of the JG strain on $4 \mathrm{dpi}$ and upregulated on 2 and $6 \mathrm{dpi}$. There were 11 miRNAs (aae-miR-2941, aae-miR-309a, aae-miR-375, aae-miR286b, aae-miR-279, aae-miR-932-5p, aae-miR-957, aaemiR-210, aae-miR-285, aae-miR-932-3p, aae-miR-307) in the salivary gland with $|\log 2 \mathrm{FC}|>2$ expressed in both the JG and MD strains of Ae. aegypti mosquitoes (Fig. 4; Table 1). Among these 11 miRNAs, aae-miR-309a, aaemiR-286b and aae-miR-279 were upregulated in both strains; Aae-miR-307 was downregulated in both strains; and Aae-miR-932-5p, aae-miR-957, aae-miR-210, aaemiR-285 and aae-miR-932-3p were downregulated in the JG strain but upregulated in the MD strain.

(See figure on next page.)

Fig. 1 Vector competence to ZIKV in two Aedes aegypti strains. a Infection rate (IR), dissemination rate (DR), transmission rate (TR) and transmission efficiency (TE) of two Ae. aegypti strains to ZIKV. A total of 30 mosquitoes were analyzed per time point, except for 20 dpi (20 individuals). b Viral RNA copies in the midgut, salivary gland and saliva of the JG and MD Ae. aegypti strains at different time points after ZIKV infection. The line represents the median of results at each time point. c Normal C6/36 cells (i) or C6/36 cells inoculated for 7 days with saliva from JG (ii) or MD (iii) mosquito strains infected with ZIKV (×100 magnification). The results shown are combined results from three independent experiments. The IR, DR, TR and TE of the two Ae. aegypti strains were compared using Fisher's exact test. Viral titers were compared using the Mann-Whitney U-test to determine differences between the two strains at different times. Abbreviations: JG, Jiegao strain; MD, Mengding strain 

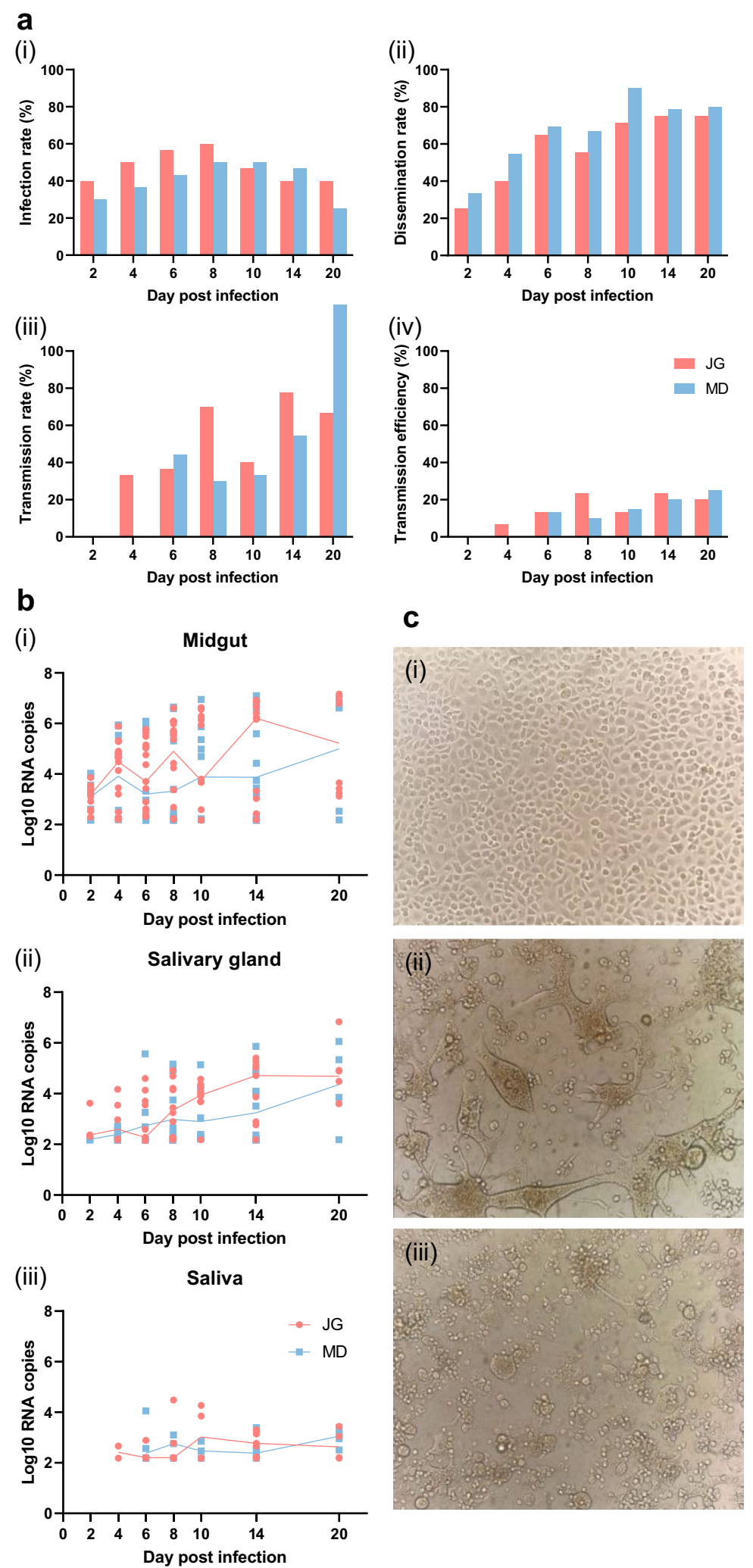

Fig. 1 (See legend on previous page.) 


\section{RT-qPCR validation of differentially expressed miRNAs}

To validate the results of deep sequencing, we determined the expression of seven miRNAs (aae-miR-989, aae-miR-2946, aae-miR-2941, aae-miR-263a-5p, aaemiR-252-5p, aae-miR-10, aae-miR-375), with RTqPCR assays on 2, 4 and 6 dpi for both the JG and MD strains (Fig. 5). The results of these assays showed that aae-miR-2946 (2 dpi), aae-miR-2941 (2 dpi), aae-miR263a-5p (4 dpi), aae-miR-252-5p ( $2 \mathrm{dpi}$ ), aae-miR-10 $(2 \mathrm{dpi})$, all from the midgut of the MD strain, exhibited inconsistencies in expression, which were seen to be depleted by RT-qPCR but enriched by RNA-Seq. While aae-miR-10 (4 dpi) from the midgut of the JG strain was seen to be upregulated with the RT-qPCR assay, deep sequencing showed the opposite result. The expression levels of other miRNAs (36 out of 42 cases) obtained by RNA-Seq and RT-qPCR were consistent, indicating that the results from RNA-Seq were reliable.

\section{Target analysis of differentially abundant miRNAs}

Elimination of duplicated miRNAs from the different groups resulted in 63 significantly differentially expressed miRNAs with $|\log 2 \mathrm{FC}|>2$. The targets of these miRNAs were predicted using the online software miRanda and RNAhybrid. These miRNAs had 4369 targets (Additional file 4: Table S4). The potential biological functions of these target genes were then explored usinge GO and KEGG functional enrichments.

In the GO function analysis processes, the biological functions related to the targets of the significantly differentially expressed miRNAs mainly included metabolic processes, cellular processes, membrane and membrane parts, catalytic activity and binding (Fig. 6). In addition, these targets may involve in the numerous KEGG pathway, such as facilitated glucose transporter, contactin-associated protein-like 2, lectin, mannosebinding 2, acetyl-CoA carboxylase/biotin carboxylase 1 , bromodomain adjacent to zinc finger domain protein 2A and peroxidase (Additional file 4: Table S4).

\section{Discussion}

A previous study demonstrated vertical transmission of ZIKV by Ae. aegypti mosquitoes from Jiegao and Mengding [25]. However, arbovirus infection of human beings mainly depends on horizontal transmission by mosquitoes. In the present study, we investigated the vector competence of these two Ae. aegypti mosquito strains to ZIKV with the aim to evaluate the risk of a ZIKA epidemic in the Jiegao and Mengding port cities of Yunan Province. We observed similar IR, DR and TR for the JG and MD strains under the same experiment condition, as well as the same propagation speed of the virus in these mosquitoes. ZIKV RNA was detected in the midgut and salivary gland of the JG and MD strains as early as $2 \mathrm{dpi}$, which is similar to results reported by Li et al. [30]; but the IR in these two tissues of the JG and MD strains were lower than those reported in the Ruili and Haikou strains by Li et al., possible due to the relative lower virus titer in the blood meal used in the latter study [30]. In our study, viral RNA was detected in the saliva of the two mosquito strains on 4 and 6 dpi, respectively. A previous study found that vector competence for DENV may be related to the genetic background of the mosquito, such as genes associated with the midgut escape barrier and, in particular, the quantitative trait loci (QTL) on chromosome III [31]. Therefore, we assume that the difference in arbovirus transmission competence between the two Ae. aegypti stains used in our study might be related to their different genetic backgrounds. CPE was observed on $7 \mathrm{dpi}$ following inoculation of saliva samples collected from the JG and MD Ae. aegypti to C6/36 cells, which demonstrated infectious activity of ZIKV in the mosquito saliva. Due to the high vector competence of these two Ae. aegypti strains to ZIKV, our study of interactions between ZIKV and the mosquitoes will facilitate the development of prevention strategies to ZIKV. Therefore, we obtained small RNA profiles in the midgut and salivary gland of ZIKV-infected and non-infected Ae. aegypti mosquitoes through high-throughput sequencing and screened out the differently expressed miRNAs, which will provide valuable information for future studies on the molecular interactions between ZIKV and mosquitoes.

miRNAs play an essential role in host resistance to infections [32], and their expression levels in mosquitoes fluctuate in accordance with infections by Plasmodium parasites and viruses [33]. In our study, small RNA library analysis of ZIKV-infected Ae. aegypti midgut and salivary gland tissues identified 87 differentially regulated miRNAs for the JG strain and 216 differentially regulated miRNAs for the MD strain at different time points

(See figure on next page.)

Fig. 2 The length distribution of small RNAs from different samples. Mosquitoes of the two strains (J, Jiego strain; M, Mengding strain) fed either a ZIKA-infected blood meal (zikv group) or a non-infected blood meal [control group (c)] were dissected at the indicated time points [2, 4, 6 days after being fed the blood meal $(2 \mathrm{~d}, 4 \mathrm{~d}, 6 \mathrm{~d}$, respectively)]. Different tissues ( $\mathrm{m}$, midgut; sg, salivary gland) were collected for RNA extraction and sequencing. Color-coded stacked bars are designated into four segments indicating the strain (J or M), the number of dpi ( $2 d, 4 d, 6 d)$, tissue type (m or sg) and experimental group (zikv or c) 

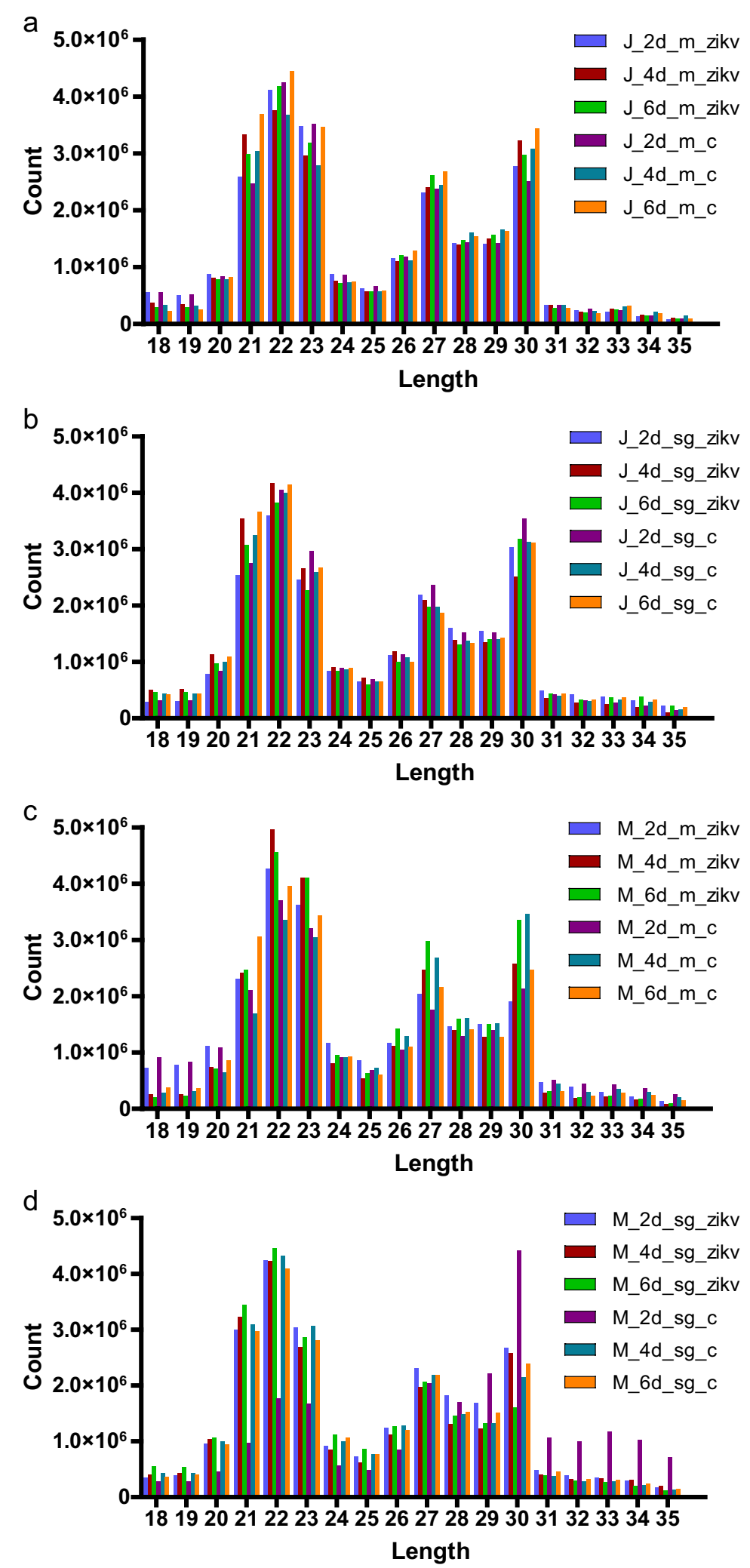

Fig. 2 (See legend on previous page.) 
Zhu et al. Parasites Vectors $\quad$ (2021) 14:504

Page 9 of 15

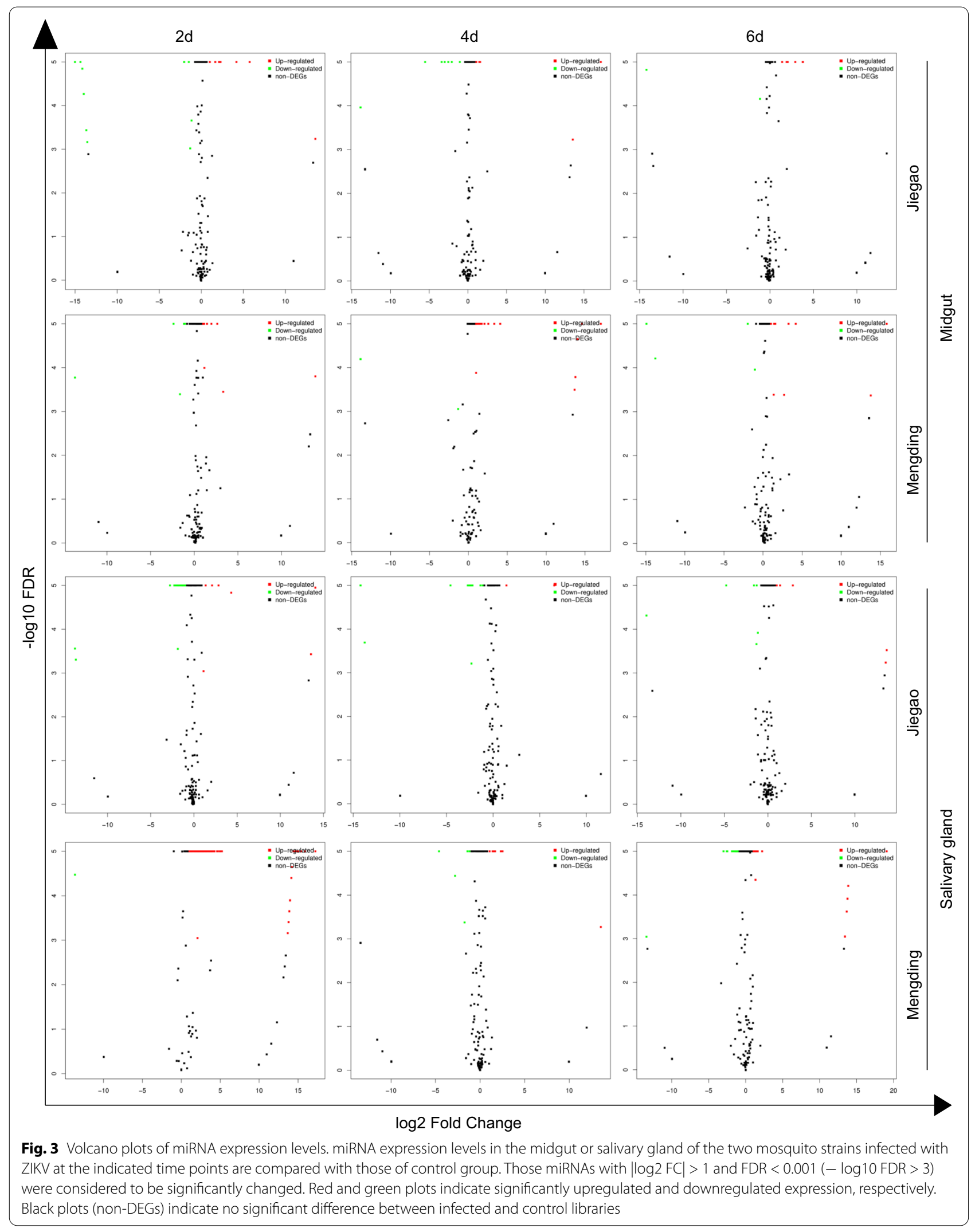




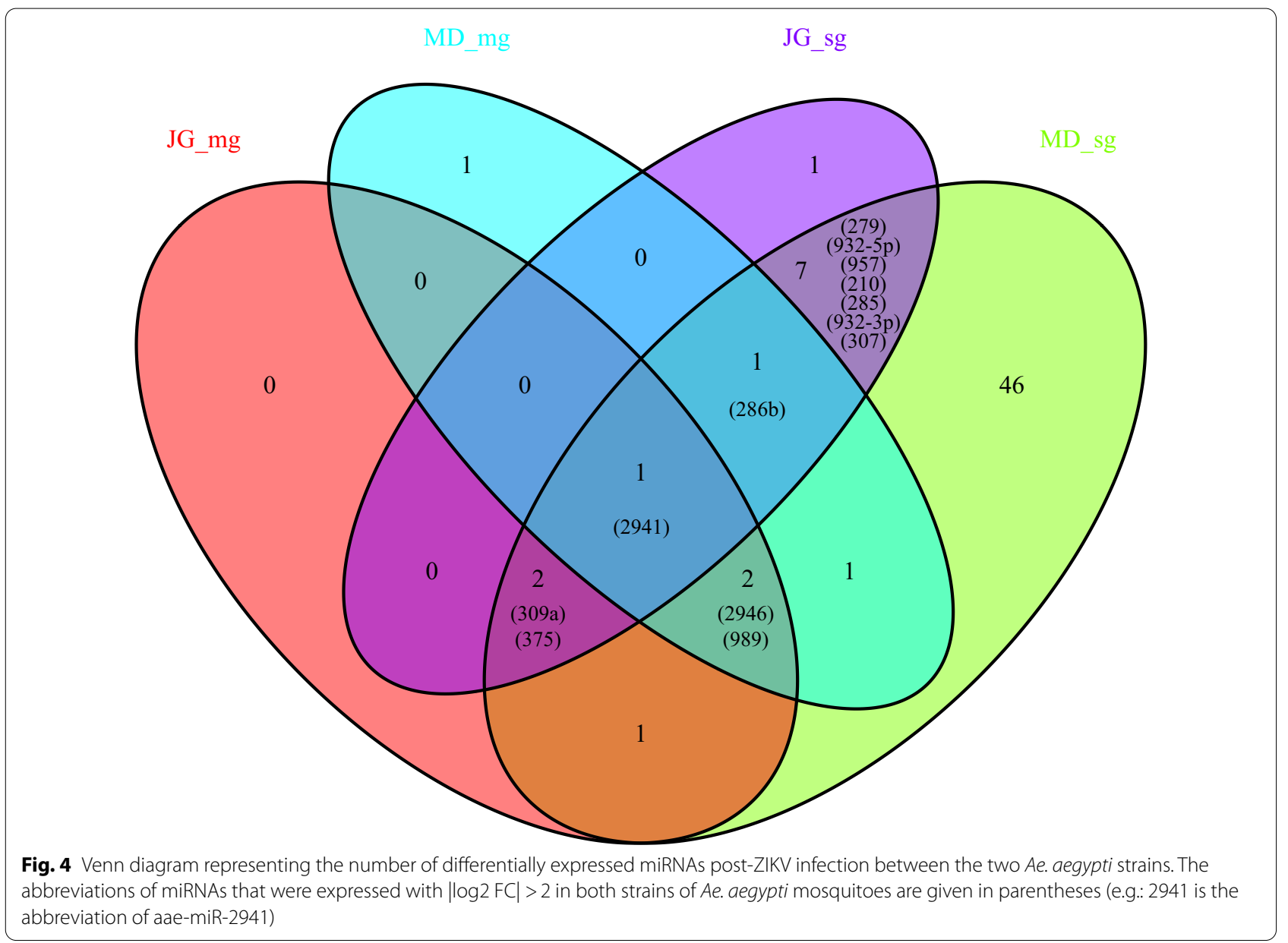

Table 1 miRNA regulation in different tissues of Ae. aegypti

\begin{tabular}{|c|c|c|c|c|c|c|c|c|c|c|c|c|}
\hline \multirow{3}{*}{ miRNAs } & \multicolumn{6}{|c|}{ Midgut } & \multicolumn{6}{|c|}{ Salivary gland } \\
\hline & \multicolumn{3}{|c|}{ JG strain } & \multicolumn{3}{|c|}{ MD strain } & \multicolumn{3}{|c|}{ JG strain } & \multicolumn{3}{|c|}{ MD strain } \\
\hline & 2dpi & 4dpi & 6dpi & 2dpi & 4dpi & 6dpi & 2dpi & 4dpi & 6dpi & 2 dpi & 4dpi & 6dpi \\
\hline aae-miR-2946 & $\mathrm{Up}$ & Down & $\mathrm{Up}$ & & Up & Up & & & & $\mathrm{Up}$ & & Down \\
\hline aae-miR-989 & Up & Down & Up & & & Up & & & & Up & & \\
\hline aae-miR-2941 & Up & Down & Up & & Up & & Down & & & $\mathrm{Up}$ & & Down \\
\hline aae-miR-309a & Up & Down & & & & & Up & & & $\mathrm{Up}$ & & \\
\hline aae-miR-375 & Down & Down & & & & & & Down & Down & Up & Down & \\
\hline aae-miR-286b & & & & Up & & & Up & & & Up & & \\
\hline aae-miR-279 & & & & & & & Up & & & Up & & \\
\hline aae-miR-932-5p & & & & & & & & Down & & $\mathrm{Up}$ & & \\
\hline aae-miR-957 & & & & & & & & Down & & Up & & \\
\hline aae-miR-210 & & & & & & & & Down & & Up & & \\
\hline aae-miR-285 & & & & & & & & Down & & Up & & Up \\
\hline aae-miR-932-3p & & & & & & & & Down & & & Up & \\
\hline aae-miR-307 & & & & & & & & Down & & & Down & \\
\hline
\end{tabular}

Down, Downregulated; Up, upregulated 

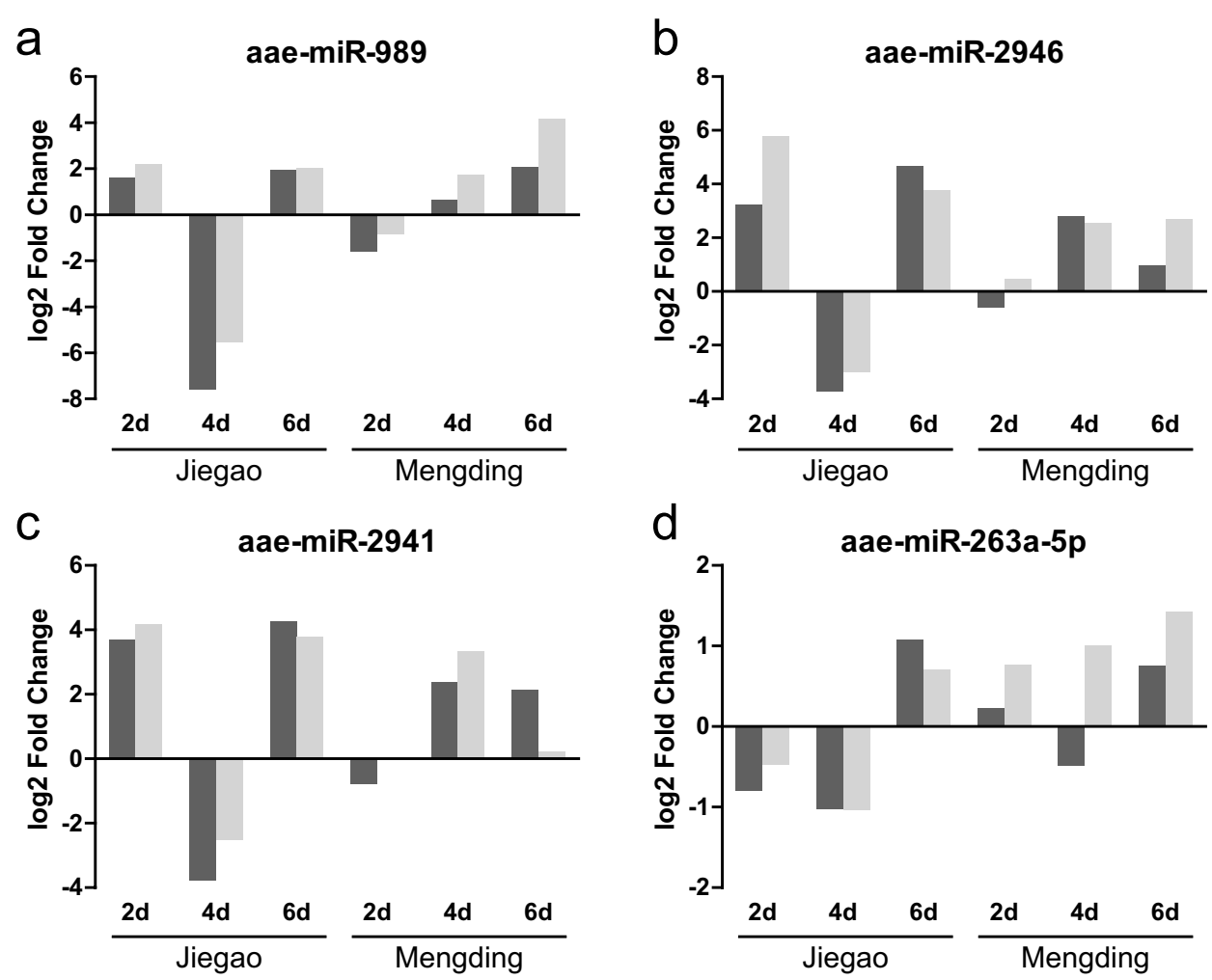

d aae-miR-263a-5p
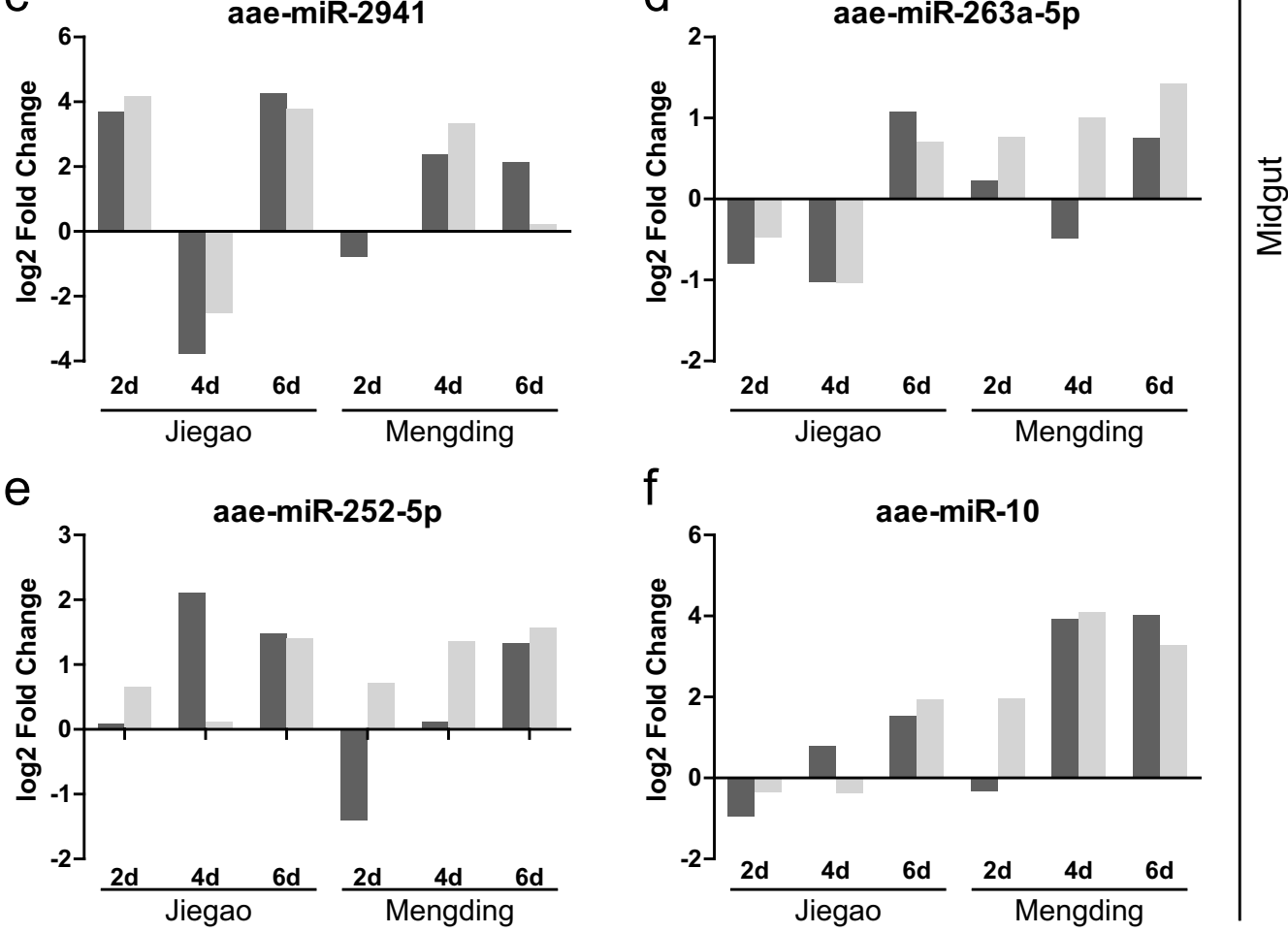

RT-qPCR assay
Deep sequencing

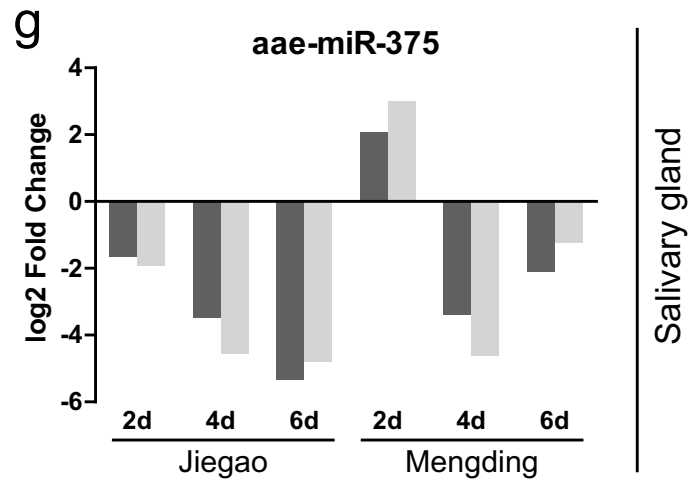

Fig. 5 RT-qPCR verification of differentially expressed miRNAs from RNA-Seq. miRNA expression in the midgut (a-f) and salivary gland ( $\mathbf{g}$ ) of the JG or MD Ae. aegypti strains infected with ZIKV relative to the uninfected group at the indicated time point is shown. For the RT-qPCR assay, fold changes are averages of three technical replicates 


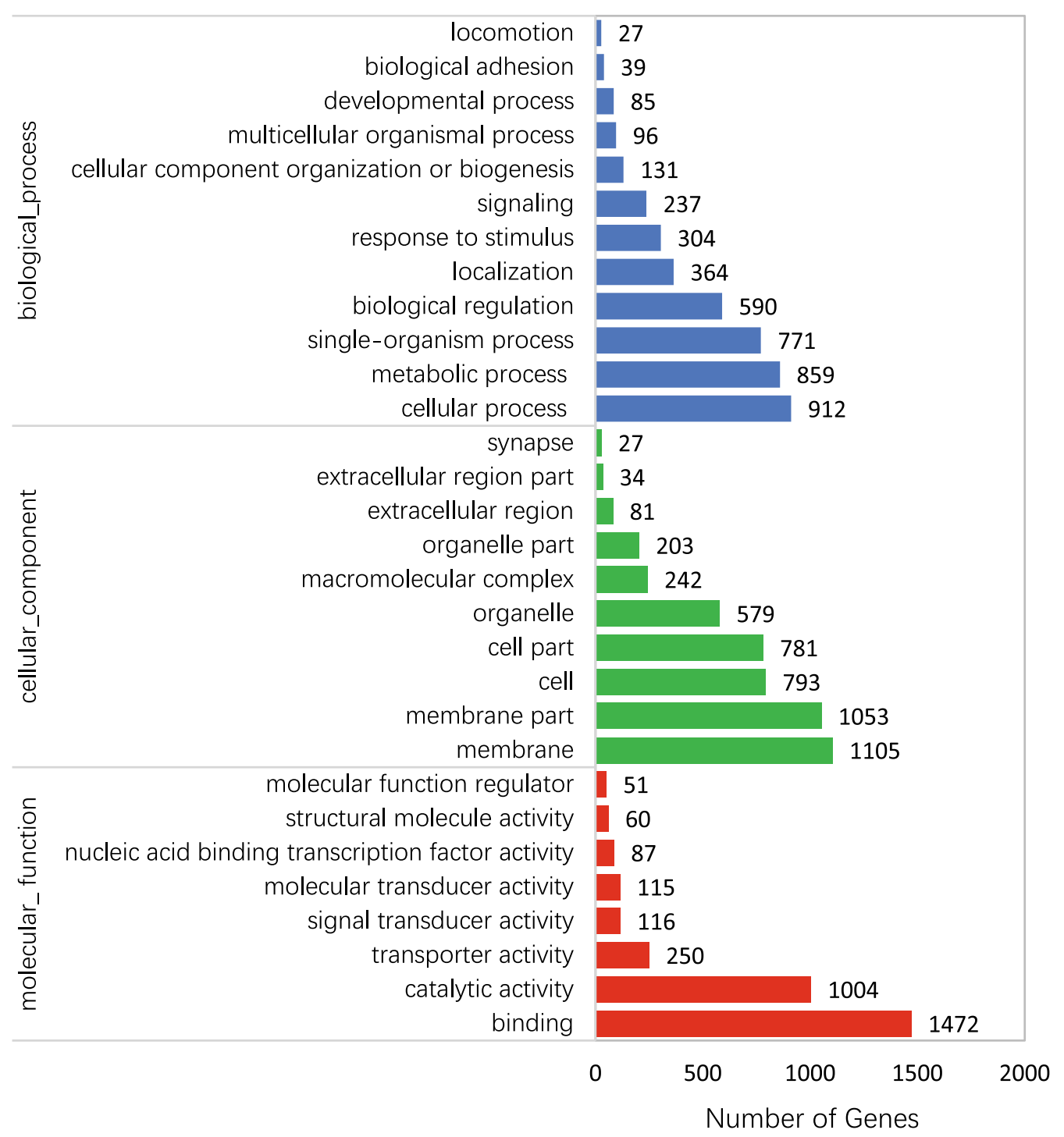

Fig. $6 \mathrm{GO}$ analysis on target genes of differently expressed miRNAs. Blue, green and red bars indicate biological processes, cellular components and molecular functions, respectively

compared with the non-infected controls. Moreover, we observed the most significant differential expressions in miRNA profile in the 2-dpi libraries of both tissues from the two strains. A similar observation was reported by Saldaña et al. who used RNA extracted from whole mosquitoes for miRNA sequencing [18]. These authors reported that miRNA modulation in ZIKV-infected Ae. aegypti was also prominent in the early phase of $2 \mathrm{dpi}$, but they found fewer changes in the miRNA profile in the mosquitoes on day 14, with the highest viral load at this time point [18]. In another study, when Ae. aegypti was infected with the Ross River virus, a similar result was observed on $2 \mathrm{dpi}$ [34]. These results highlight the potential importance of this period ( $2 \mathrm{dpi}$ ) during arbovirus infection and indicate that there is no positive correlation between changes in miRNA expression and viral load.

In the present study, further screening resulted in 27 and 74 miRNAs being selected from the miRNA expression profiles of ZIKV-infected and non-infected midgut and salivary gland tissues from JG and MD strain, respectively. Variability in transcriptomes was found between these different strains of Ae. aegypti, which indicates the need for caution in making generalizations about individual gene expression profiles across different strains of Ae. aegypti [35]. Thus, we hypothesize that the difference 
in the miRNA expression profile may be related to the different genetic backgrounds of the mosquitoes. The most significantly differentially expressed miRNAs of the midgut and salivary gland tissues from the two $A e$. aegypti strains found in this study are aae-miR-2946 $(\log 2 \mathrm{FC}=5.75$, midgut, JG strain), aae-miR-989 ( $\log 2$ $\mathrm{FC}=-5.53$, midgut, JG strain), aae-miR-286b (log2 $\mathrm{FC}=4.32$, salivary gland, JG strain), aae-miR-375 ( $\log 2$ $\mathrm{FC}=-4.79$, salivary gland, JG strain); aae-miR-989 ( $\log 2 \mathrm{FC}=4.18$, midgut, MD strain), aae-miR-957 $(\log 2$ $\mathrm{FC}=5.22$, salivary gland, $\mathrm{MD}$ strain) and ae-miR-375 $(\log 2 \mathrm{FC}=-4.62$, salivary gland, MD strain). In other studies, those miRNAs were also reported after arboviruses infection. For example, aae-miR-375 was found to be mostly depleted after ZIKV infection [18], and it has also been shown to enhance DENV serotype 2 infection in an Ae. aegypti cell line [22]. In our study, aae-miR-375 showed the highest depletion in the salivary gland of two strains. Therefore, it will be interesting to experimentally test the function of this miRNA on ZIKV infection. Similarly, in the present study, the maximum FC was found in aae-miR-989 in the midgut. In comparison, Winter et al. reported that miR-989 also showed significant differential expression $(\log 2 \mathrm{FC}=4)$ in Plasmodium-infected Anopheles gambiae and considered it to be a mosquito midgut-specific miRNA [36], whereas other studies found miR-989 to be rarely expressed in the midgut tissue of Anopheles stephensi [37] and exclusively expressed in the ovary of An. gambiae [38]. These contradictory conclusions may be due to the different mosquito species and viruses used in the different studies. In addition, aaemiR-989 is considered to be an important miRNA targeting mosquito immunity $[39,40]$. Aae-miR-286b was downregulated in a study involving C6/36 Ae. albopictus cell line infected with DENV-2 [41]. In the present study, we report for the first time that aae-miR-2946 and aaemiR-957 showed significant differential expression after ZIKV blood meal in Ae. aegypti. To our knowledge, there have been no reports to date on the expression of these two miRNAs after ZIKV infection. We also found that aae-miR-2946, aae-miR-2941, aae-miR-989 and aae-miR286b showed significant differential expression between both strains of the Ae. aegypti strains after the virus blood meal.

In terms of $\mathrm{GO}$ function analysis processes, the targets of the significantly differentially expressed miRNAs may be involved in metabolic processes, cellular processes, membrane and membrane parts and catalytic activity and binding, which indicates that the ZIKVvector interaction is a complicated and highly regulated process. Recent research has shown that the metabolic conditions in the mosquito tissues play a critical role in the process of virus infection and replication and partially regulate virus transmission. Moreover, it has been demonstrated that ZIKV infection results in changes to the cellular metabolic environment, such as a significant enrichment of inosine and pseudo-uridine $(\Psi)$ levels which may be related to RNA editing activity [42]. Therefore, future mechanistic studies can provide a new perspective to our understanding of the interactions between the ZIKV and its vector host.

\section{Conclusions}

Both the JG and MD Ae. aegypti strains possess a strong vector competence to ZIKV. In this study, we compared miRNA profiles in the midgut and salivary gland of ZIKV-infected and non-infected Ae. aegypti mosquitoes. Differently expressed miRNAs were screened, which will provide valuable information for investigating the molecular mechanisms that influence the vector competence of mosquitoes. miRNAs regulate gene expression mainly by targeting cognate mRNAs for cleavage or translational repression. In a future study, we will seek to selected these targets of miRNAs by transcriptomics analysis or mRNA-Seq and validate the function in the regulation of mosquito competence, which will help to provide more efficient vector prevention strategies.

\section{Abbreviations \\ CDNA: Complementary DNA; CHIKV: Chikungunya virus; CPE: Cytopathic effect; DENV: Dengue virus; DMEM: Dulbecco's modified Eagle's medium; dpi: Days post-infection; DR: Dissemination rate; FBS: Fetal bovine serum; FC: Fold change; FDR: False discovery rate; GO: Gene Ontology; IR: Infection rate; JG: Jiegao; KEGG: Kyoto Encyclopedia of Genes and Genomes; MD: Meng- ding; miRNA: MicroRNA; PAGE: Polyacrylamide gel electrophoresis; piRNA: P-element-induced wimpy testis-interacting RNA; QTL: Quantitative trait loci; RISC: RNA-induced silencing complex; RNAi: RNA interference; RNA-seg: RNA sequencing; ROS: Reactive oxygen species; rRNA: Ribosomal RNA; RT-PCR: reverse transcriptase-PCR; RT-qPCR: Reverse transcription-quantitative PCR; scRNA: Small cytoplasmic RNA; siRNA: Small interfering RNA; snoRNA: Small nucleolar RNA; snRNA: Small nuclear RNA; TE: Transmission efficiency; TR: Transmission rate; tRNA: Transfer RNA; ZIKV: Zika virus.}

\section{Supplementary Information}

The online version contains supplementary material available at https://doi. org/10.1186/s13071-021-05007-7.

Additional file 1: Table S1. Primers used for reverse transcription and quantitative PCR.

Additional file 2: Table S2. High-throughput sequencing profile of Small RNA.

Additional file 3: Table S3. The differentially expressed miRNAs based on small RNA-seq ( $|\log 2 \mathrm{FC}|>2)$.

Additional file 4: Table S4. Target genes predicted from differentially expressed miRNAs ( $|\log 2 \mathrm{FC}|>2$ ).

\section{Acknowledgements}

Not applicable. 


\section{Authors' contributions}

XXG and TYZ designed the study. CLZ, YTJ, QHZ, JG and CJL performed the laboratory experiments. CXL, YDD and DX provided the materials. CLZ, YTJ, HDZ and TZ analyzed and interpreted the data. CLZ wrote the manuscript. YTJ, XXG and TYZ contributed to manuscript revision. All authors read and approved the final manuscript.

\section{Funding}

This work was funded by grants from the Infective Diseases Prevention and Cure Project of China (No. 2017ZX10303404).

\section{Availability of data and materials}

All data generated or analyzed during this study are included in this published article and its additional files.

\section{Declarations}

\section{Ethics approval and consent to participate}

The animal studies were carried out in strict accordance with the recommendations in the Guide for the Care and Use of Laboratory Animals and were approved by the IACUC of the State Key Laboratory of Pathogen and Biosecurity, Beijing Institute of Microbiology and Epidemiology (permit number: IACUC-IME-2017-016).

\section{Consent for publication}

Not applicable.

\section{Competing interests}

The authors declare that they have no competing interests.

\section{Author details}

${ }^{1}$ Department of Vector Biology and Control, State Key Laboratory of Pathogen and Biosecurity, Beijing Key Laboratory of Vector Borne and Natural Focus Infectious Diseases, Beijing Institute of Microbiology and Epidemiology, Beijing 100071, China. ${ }^{2}$ Department of Clinical Laboratory, Guangxi International Zhuang Medicine Hospital, Nanning 530201, Guangxi, China.

Received: 6 April 2021 Accepted: 9 September 2021

Published online: 27 September 2021

\section{References}

1. Atif M, Azeem M, Sarwar MR, Bashir A. Zika virus disease: a current review of the literature. Infection. 2016;44(6):695-705.

2. Dick GW, Kitchen SF, Haddow AJ. Zika virus. I. Isolations and serological specificity. Trans R Soc Trop Med Hyg. 1952;46(5):509-20.

3. Gubler DJ, Vasilakis N, Musso D. History and emergence of Zika virus. J Infect Dis. 2017;216(suppl_10):S860-7.

4. Campos GS, Bandeira AC, Sardi SI. Zika virus outbreak, Bahia, Brazil. Emerg Infect Dis. 2015;21(10):1885-6.

5. Zanluca C, Melo VC, Mosimann AL, Santos Gl, Santos CN, Luz K. First report of autochthonous transmission of Zika virus in Brazil. Mem Inst Oswaldo Cruz. 2015;110(4):569-72.

6. Baud D, Gubler DJ, Schaub B, Lanteri MC, Musso D. An update on Zika virus infection. Lancet. 2017;390(10107):2099-109.

7. Pierson TC, Diamond MS. The emergence of Zika virus and its new clinical syndromes. Nature. 2018;560(7720):573-81.

8. Beaty BJ. Genetic manipulation of vectors: a potential novel approach for control of vector-borne diseases. Proc Natl Acad Sci USA. 2000;97(19):10295-7.

9. Aksoy S, Maudlin I, Dale C, Robinson AS, O'Neill SL. Prospects for control of African trypanosomiasis by tsetse vector manipulation. Trends Parasitol. 2001;17(1):29-35.

10. Alphey L, Beard CB, Billingsley P, Coetzee M, Crisanti A, Curtis C, et al. Malaria control with genetically manipulated insect vectors. Science. 2002;298(5591):119-21.

11. Franz AW, Kantor AM, Passarelli AL, Clem RJ. Tissue barriers to arbovirus infection in mosquitoes. Viruses. 2015;7(7):3741-67.
12. Oliveira JHM, Talyuli OAC, Goncalves RLS, Paiva-Silva GO, Sorgine MHF, Alvarenga $\mathrm{PH}$, et al. Catalase protects Aedes aegypti from oxidative stress and increases midgut infection prevalence of Dengue but not Zika. PLoS Negl Trop Dis. 2017;11(4):e0005525.

13. Ramirez JL, Dimopoulos $\mathrm{G}$. The Toll immune signaling pathway control conserved anti-dengue defenses across diverse Ae. aegypti strains and against multiple dengue virus serotypes. Dev Comp Immunol. 2010;34(6):625-9.

14. McFarlane M, Arias-Goeta C, Martin E, O'Hara Z, Lulla A, Mousson $L$, et al. Characterization of Aedes aegypti innate-immune pathways that limit Chikungunya virus replication. PLoS Negl Trop Dis. 2014;8(7):e2994

15. Jupatanakul N, Sim S, Anglero-Rodriguez YI, Souza-Neto J, Das S, Poti KE, et al. Engineered Aedes aegypti JAK/STAT pathway-mediated immunity to dengue virus. PLoS Negl Trop Dis. 2017;11(1):e0005187.

16. Souza-Neto JA, Sim S, Dimopoulos G. An evolutionary conserved function of the JAK-STAT pathway in anti-dengue defense. Proc Natl Acad Sci USA. 2009;106(42):17841-6.

17. Ghildiyal M, Zamore PD. Small silencing RNAs: an expanding universe. Nat Rev Genet. 2009;10(2):94-108.

18. Saldana MA, Etebari K, Hart CE, Widen SG, Wood TG, Thangamani $S$, et al. Zika virus alters the microRNA expression profile and elicits an RNAi response in Aedes aegypti mosquitoes. PLoS Negl Trop Dis. 2017:11(7):e0005760.

19. Kumar A, Srivastava P, Sirisena P, Dubey SK, Kumar R, Shrinet J, et al. Mosquito innate immunity. Insects. 2018;9:3.

20. Campbell CL, Harrison T, Hess AM, Ebel GD. MicroRNA levels are modulated in Aedes aegypti after exposure to Dengue-2. Insect Mol Biol. 2014;23(1):132-9.

21. Yan H, Zhou Y, Liu Y, Deng Y, Chen X. miR-252 of the Asian tiger mosquito Aedes albopictus regulates dengue virus replication by suppressing the expression of the dengue virus envelope protein. J Med Virol. 2014;86(8):1428-36.

22. Hussain M, Walker T, O'Neill SL, Asgari S. Blood meal induced microRNA regulates development and immune associated genes in the Dengue mosquito vector, Aedes aegypti. Insect Biochem Mol Biol. 2013;43(2):146-52.

23. Shi QM, Zhang HD, Wang G, Guo XX, Xing D, Dong YD, et al. The genetic diversity and population structure of domestic Aedes aegypti (Diptera: Culicidae) in Yunnan Province, southwestern China. Parasit Vector. 2017;10:1

24. Deng YQ, Zhao H, Li XF, Zhang NN, Liu ZY, Jiang T, et al. Isolation, identification and genomic characterization of the Asian lineage Zika virus imported to China. Sci China Life Sci. 2016;59(4):428-30.

25. Zhu C, Jiang Y, Zhang Q, Gao J, Gu Z, Lan C, et al. Verticaltransmission of Zika virus by Jiegao and Mengding Aedes aegypti (Diptera: Culicidae) strains in Yunnan Province in China. Vector Borne Zoonotic Dis. 2020;20(9):664-9.

26. Dubrulle M, Mousson L, Moutailler S, Vazeille M, Failloux AB. Chikungunya virus and Aedes mosquitoes: saliva is infectious as soon as two days after oral infection. PLoS ONE. 2009;4(6):e5895.

27. Calabrese JM, Seila AC, Yeo GW, Sharp PA. RNA sequence analysis defines Dicer's role in mouse embryonic stem cells. Proc Natl Acad Sci USA. 2007;104(46):18097-102.

28. Audic S, Claverie JM. The significance of digital gene expression profiles. Genome Res. 1997;7(10):986-95.

29. Livak KJ, Schmittgen TD. Analysis of relative gene expression data using real-time quantitative PCR and the 2(-Delta Delta $C(T)$ ) method. Methods. 2001;25(4):402-8.

30. Li CX, Guo XX, Deng YQ, Xing D, Sun AJ, Liu QM, et al. Vector competence and transovarial transmission of two Aedes aegypti strains to Zika virus. Emerg Microbes Infect. 2017;6(4):e23.

31. Tabachnick WJ. Nature, nurture and evolution of intra-species variation in mosquito arbovirus transmission competence. Int J Environ Res Public Health. 2013;10(1):249-77.

32. Samuel GH, Adelman ZN, Myles KM. Antiviral immunity and virusmediated antagonism in disease vector mosquitoes. Trends Microbiol. 2018;26(5):447-61.

33. Feng $X$, Zhou S, Wang J, Hu W. microRNA profiles and functions in mosquitoes. PLoS Negl Trop Dis. 2018;12(5):e0006463. 
34. Sinclair JB, Asgari S. Ross river virus provokes differentially expressed microRNA and RNA interference responses in Aedes aegypti mosquitoes. Viruses. 2020;12:695.

35. Bonizzoni M, Dunn WA, Campbell CL, Olson KE, Marinotti O, James AA. Strain variation in the transcriptome of the dengue fever vector, Aedes aegypti. G3. 2012;2(1):103-14.

36. Winter F, Edaye S, Huttenhofer A, Brunel C. Anopheles gambiae miRNAs as actors of defence reaction against Plasmodium invasion. Nucleic Acids Res. 2007:35(20):6953-62.

37. Mead EA, Tu Z. Cloning, characterization, and expression of microRNAs from the Asian malaria mosquito, Anopheles stephensi. BMC Genomics. 2008;9:244.

38. Lampe L, Levashina EA. MicroRNA tissue atlas of the malaria mosquito Anopheles gambiae. G3. 2018;8(1):185-93.

39. Hussain M, Frentiu FD, Moreira LA, O'Neill SL, Asgari S. Wolbachia uses host microRNAs to manipulate host gene expression and facilitate colonization of the dengue vector Aedes aegypti. Proc Natl Acad Sci USA 2011;108(22):9250-5.
40. Jain S, Rana V, Tridibes A, Sunil S, Bhatnagar RK. Dynamic expression of miRNAs across immature and adult stages of the malaria mosquito Anopheles stephensi. Parasit Vectors. 2015;8:179.

41. Avila-Bonilla RG, Yocupicio-Monroy M, Marchat LA, De Nova-Ocampo MA, Del Angel RM, Salas-Benito JS. Analysis of the miRNA profile in C6/36 cells persistently infected with dengue virus type 2 . Virus Res. 2017;232:139-51.

42. Onyango MG, Attardo GM, Kelly ET, Bialosuknia SM, Stout J, Banker E, et al. Zika virus infection results in biochemical changes associated with RNA editing, inflammatory and antiviral responses in Aedes albopictus. Front Microbiol. 2020;11:559035.

\section{Publisher's Note}

Springer Nature remains neutral with regard to jurisdictional claims in published maps and institutional affiliations.
Ready to submit your research? Choose BMC and benefit from:

- fast, convenient online submission

- thorough peer review by experienced researchers in your field

- rapid publication on acceptance

- support for research data, including large and complex data types

- gold Open Access which fosters wider collaboration and increased citations

- maximum visibility for your research: over $100 \mathrm{M}$ website views per year

At BMC, research is always in progress.

Learn more biomedcentral.com/submissions 